\title{
Monte Carlo Analysis of Pion Contribution to Absorbed Dose from Galactic Cosmic Rays
}

S. K. Aghara ${ }^{\mathrm{a} *}$, S. R. Blattnig ${ }^{\mathrm{b}}$, J. W. Norbury ${ }^{\mathrm{b}}$ and R. C. Singleterry ${ }^{\mathrm{b}}$

a Prairie View A \& $M$ University, Prairie View, Texas 77446

b NASA Langley Research Center, Hampton, Virginia 23681

*Author to whom correspondence should be addressed at Prairie View A \& M University, P.O. Box 519, MS 2505, Prairie View, Texas 77446; email: Sukesh.K.Aghara@nasa.gov 


\title{
S. K. Aghara, S. R. Blattnig, J. W. Norbury and R. C. Singleterry, Monte Carlo Analysis of Pion Contribution to Absorbed Dose from Galactic Cosmic Rays. Nucl. Instrum. Meth. B, (2008).
}

\begin{abstract}
\end{abstract}
Accurate knowledge of the physics of interaction, particle production and transport is necessary to estimate the radiation damage to equipment used on spacecraft and the biological effects of space radiation. For long duration astronaut missions, both on the International Space Station and the planned manned missions to Moon and Mars, the shielding strategy must include a comprehensive knowledge of the secondary radiation environment. The distribution of absorbed dose and dose equivalent is a function of the type, energy and population of these secondary products. Galactic cosmic rays (GCR) comprised of protons and heavier nuclei have energies from a few $\mathrm{MeV}$ per nucleon to the $\mathrm{ZeV}$ region, with the spectra reaching flux maxima in the hundreds of $\mathrm{MeV}$ range. Therefore, the $\mathrm{MeV}-\mathrm{GeV}$ region is most important for space radiation. Coincidentally, the pion production energy threshold is about $280 \mathrm{MeV}$. The question naturally arises as to how important these particles are with respect to space radiation problems. The space radiation transport code, HZETRN (High charge (Z) and Energy TRaNsport), currently used by NASA, performs neutron, proton and heavy ion transport explicitly, but it does not take into account the production and transport of mesons, photons and leptons. In this paper, we present results from the Monte Carlo code MCNPX (Monte Carlo NParticle eXtended), showing the effect of leptons and mesons when they are produced and transported in a GCR environment. 


\section{INTRODUCTION}

Protecting astronauts from the harmful effects of space radiation is an important priority for human space flight $[1,2]$. The shielding strategies for an extended stay in space must include knowledge of the internal radiation environment induced by the primary, external radiation. Primary radiation particles undergo atomic and nuclear interactions as they pass through matter, thereby producing secondary particles. The radiation downstream consists of modified particle fluxes. The interactions of various radiations with matter are unique and determine their depth of penetration. This consequently impacts the type and amount of shielding needed for radiation protection. Hence, for optimal radiation shield design, a complete characterization of the secondary radiation products is necessary.

The space radiation environment is comprised of energetic particles produced from three sources, each with a characteristic spectrum. Firstly, solar particle events (SPE) consist primarily of protons emitted from the Sun during coronal mass ejections and solar flares. These events are rare, but when they occur, they can inflict a potentially lethal dose of radiation to astronauts if no protective measures are undertaken. They are also of great concern for the stability of electronic devices. Energies often reach hundreds of $\mathrm{MeV}$ and can even extend into the GeV region. Secondly, Galactic cosmic rays (GCR) are protons and heavier nuclei thought to be emitted from supernovae explosions within the Milky Way galaxy and accelerated to the vicinity of the Solar system. The GCR particles have energies from a few $\mathrm{MeV}$ per nucleon up to the $\mathrm{ZeV}$ region (Zetta eV = $10^{21} \mathrm{eV}$ ). The GCR spectra reach flux maxima in the hundreds of $\mathrm{MeV}$ range and so the $\mathrm{MeV}-\mathrm{GeV}$ region is most important for space radiation. Radiation dose from nuclei is approximately proportional to $Z^{2}$, where $\mathrm{Z}$ is the ion charge, and so GCR ions from Hydrogen to Nickel are of most concern [1]. Beyond Nickel, the particle flux is much 
smaller and generally ignored. Thirdly, the geomagnetically trapped particles are protons and electrons confined by the magnetic field of Earth. There are two distinct regions called the inner and outer Van Allen radiation belts. Protons and electrons are found in both belts. The proton energies range up to $100 \mathrm{MeV}$ and beyond. The electron energies range from $100 \mathrm{keV}$ and beyond. For the purpose of this study, we confine our external radiation environment to GCR. Parallel studies are underway for the SPE and trapped belt environments.

A comprehensive radiation shielding design study requires characterizing the primary radiation and the resulting secondary radiation. HZETRN (High Z and Energy TRaNsport, where $\mathrm{Z}$ is the charge) is a space radiation transport code currently used by NASA to characterize the space radiation environment inside spacecraft for human exposures $[2,3,4]$. It performs neutron, proton and heavy ion transport, but it does not take into

account the production and transport of mesons, photons and leptons $[4,5]$. The question naturally arises as to how important these particles are with respect to space radiation problems. Compared to heavier mesons, pions are the lightest meson and are therefore produced more copiously in cosmic ray interactions. Subsequently, through decay or other interactions, pions produce hadrons, photons and leptons. In this paper, we present the results of MCNPX (Monte Carlo N-Particle eXtended) simulations that quantify the differences in absorbed dose when pions are produced and transported in a GCR environment.

\section{HADRON PHYSICS}

We begin with a review of the relevant physics pertaining to pion production. The Standard Model of particle physics [6] describes our universe in terms of fundamental 
particles, called quarks and leptons, which interact via the electromagnetic, strong, or weak force. The interactions are mediated by photons $(\gamma)$, gluons, and $W^{ \pm}, Z^{0}$ bosons (mediators) particles. The spectrum of hadrons consists of all possible allowed combinations of bound states of quarks. These bound states occur in both ground and excited states, resulting in a large number of possible hadrons with a variety of masses. There are two classes of hadrons, called baryons (three quark) and mesons (one quark and one antiquark). The lowest mass baryon is the nucleon, which has two charge states, which are the proton $(p)$ or neutron $(n)$. The lowest mass meson is the pion, which occurs in three different charge states $\left(\pi^{ \pm}, \pi^{0}\right)$.

Cosmic ray interactions with matter include high energy proton - nucleus and nucleus nucleus collisions, whereby a nucleus may break up into its constituent pieces, producing lighter nuclei in the final state through nuclear fragmentation. Most space radiation studies include the baryons but neglect the radiation dose produced by mesons, leptons and their decay products [6]. Because the pion is the lowest mass hadron, it is the most produced particle in the nucleon - nucleon collisions that occur in cosmic ray nuclear interactions. The heavier mesons are produced in fewer numbers. The question naturally arises as to what is the contribution of these particles to space radiation. In this paper, we investigate the pion contribution to absorbed dose.

Threshold energies for several pion producing reactions in proton - proton $(p p)$ collisions are listed in Table 1 . The threshold for $\pi^{0}$ production is at a kinetic energy of $280 \mathrm{MeV}$. Double pion production begins at $592 \mathrm{MeV}$. The GCR spectra, coincidentally, reach flux maxima in the hundreds of $\mathrm{MeV}$ range, corresponding to the pion production threshold. Hence, in a GCR environment (proton peak at about $360 \mathrm{MeV}$ ), we expect to see both neutral and charged pion production. Further, we also expect to see other nucleon - nucleon reactions producing pions as listed in Table 2. 
Table 1: Kinetic energy thresholds $(\mathrm{MeV})$ for proton - proton $(p p)$ reactions. Particle symbols are proton $p$, neutron $n$, deuteron $d$ and pion $\pi$.

\begin{tabular}{lc}
\hline Final state & $\begin{array}{r}\text { Threshold } \\
(\mathrm{MeV})\end{array}$ \\
\hline & \\
\hline$p p \pi^{0}$ & 280 \\
$d \pi^{+}$ & 288 \\
$p n \pi^{+}$ & 292 \\
$p n \pi^{+} \pi^{0}$ & 592 \\
$p p \pi^{+} \pi^{-}$ & 600 \\
$p p \pi^{+} \pi^{-} \pi^{0}$ & 920 \\
& \\
\hline
\end{tabular}

Table 2: Some nucleon-nucleon reactions producing pions.

$$
\begin{array}{ll}
p+p \quad \rightarrow \quad & p+p \\
& n+p+\pi^{+} \\
& \pi^{0}+p+p \\
& \pi^{+}+p+n \\
& \pi^{+}+d \\
& \pi^{-}+p+p+\pi^{+} \\
n+n \quad \rightarrow \quad & p+n+\pi^{-} \\
& n+n \\
& \pi^{0}+n+n \\
& \pi^{+}+\pi^{-}+n+n \\
& \pi^{-}+p+n \\
& \pi^{-}+d \\
& p+n \\
& n+p \\
& \pi^{0}+p+n \\
& \pi^{0}+d \\
& \pi^{+}+n+n \\
& \pi^{-}+p+p
\end{array}
$$


Once produced, the neutral pion will decay immediately, whereas the charged pions will travel some distance before they decay. The $\pi^{0}$ decays via the electromagnetic interaction, whereas the $\pi^{ \pm}$decay via the weak interaction. The electromagnetic interaction, being stronger than the weak interaction at this energy scale, accounts for the much shorter lifetime of the $\pi^{0}$ compared to the charged particles. The interactions resulting from the pion channels will result in the production of electromagnetic particles, such as electrons, positrons, photons and muons, which are the main source of electromagnetic (EM) cascades. The pion primary decay modes and lifetimes $(\tau)$ are listed in Table 3 . In Table 3, the lifetime $\tau$ has been listed as well as the quantity $c \tau$. Both of these are given in the rest frame of the decaying particle. The quantity $c \tau$ gives an idea of how far the particle will travel before decaying. Of course, in the lab frame, which is the target frame or spacecraft wall frame, the lifetime will be longer, due to time dilation, and the distance will therefore be longer. Thus, $c \tau$ is actually a minimum distance, but it gives a rough idea of the range of a particle. In the flux versus depth and dose versus depth curves, we expect to see the effect of the neutral pion decay through the build up of photons.

Table 3: Primary decay modes [6]. The mean lifetime is given by the symbol $\tau$ and $c \tau$ is the speed of light multiplied by the mean lifetime. Particle symbols are pion $\pi$, muon $\mu$, photon $\gamma$ and neutrino $\nu$.

\begin{tabular}{ccccc}
\hline Particle & $\begin{array}{c}\text { Rest Mass } \\
\left(\mathrm{MeV} / \mathrm{c}^{2}\right)\end{array}$ & $\begin{array}{c}\text { Decay } \\
\text { Mode }\end{array}$ & $\begin{array}{c}\tau \\
(\mathrm{sec})\end{array}$ & $\begin{array}{c}c \tau \\
(\mathrm{m})\end{array}$ \\
\hline & & & & \\
$\pi^{0}$ & 135 & $\gamma \gamma$ & $8.4 \times 10^{-17}$ & $25 \times 10^{-9}$ \\
$\pi^{+}$ & 140 & $\mu^{+} \nu_{\mu}$ & $2.6 \times 10^{-8}$ & 7.8 \\
$\pi^{-}$ & 140 & $\mu^{-} \bar{\nu}_{\mu}$ & $2.6 \times 10^{-8}$ & 7.8 \\
$\mu^{+}$ & 106 & $e^{+} \nu_{e} \bar{\nu}_{\mu}$ & $2.2 \times 10^{-6}$ & 660 \\
$\mu^{-}$ & 106 & $e^{-} \bar{\nu}_{e} \nu_{\mu}$ & $2.2 \times 10^{-6}$ & 660 \\
& & & & \\
\hline
\end{tabular}


Since the lifetime of charged pions is large, they will interact with other nucleons before they decay, producing pions and nucleons in the final state. Examples of such reactions are listed in Table 4 . These pion interactions will impact the overall flux of light ions and baryons. Hence, in a GCR transport calculation, using a transport code that includes hadron (baryons and mesons) and lepton calculations explicitly, we expect to see production of EM particles and EM cascades.

Table 4: Charged pion - nucleon reactions.

$$
\begin{array}{rlrl}
\pi^{+}+p \rightarrow & p+\pi^{+} & \pi^{+}+n \rightarrow & p+\pi^{0} \\
& n+\pi^{+}+\pi^{+} & & n+\pi^{+} \\
& \pi^{0}+p+\pi^{+} & & \pi^{0}+p \\
& \pi^{+}+p & \pi^{+}+n \\
& \pi^{-}+p+\pi^{+}+\pi^{+} & \pi^{-}+p+\pi^{+}
\end{array}
$$

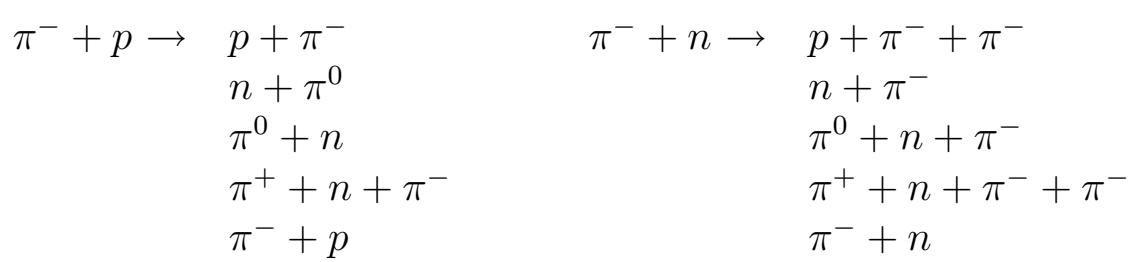

\section{RADIATION TRANSPORT}

The interactions of the incident primary radiation with the target nuclei (shielding or human tissue) result in a modified radiation field downstream. A detailed and accurate characterization of the radiation field in terms of absorbed dose $(\mathrm{D})$, dose equivalent $(\mathrm{H})$ and other end point quantities, is necessary to assess the short and long term response of biological systems and electronics to the exposed radiation. 
The primary radiation particles from a GCR spectrum travel through the spacecraft material producing secondary particles. The nuclear fragmentation processes result in a cascade of secondary products, such as leptons, baryons, mesons, photons, electrons, positrons, light $(A \leq 4)$ and heavy ions $(A>4)$. For a comprehensive transport calculation, all the secondary products should be modeled explicitly. However, a fully coupled transport calculation that includes all possible secondary products is typically not performed due to two main reasons. First, these types of calculations traditionally impose considerable penalty on time-to-solution for even simple geometries. Second, due to their lower weighting factors (quality factors), the dose equivalent contribution from leptons and mesons is perceived to be negligible, when compared with the dose contributions from light ions, heavy ions and baryons. Hence, in most transport calculations, certain production and transport channels are not explicitly defined or are turned off to speed up the calculations. In many cases, these assumptions have been valid. However, review of the particle theory suggests that for the GCR environment, the meson, lepton and photon contributions may merit further investigation.

A systematic verification and validation (V\&V) effort, including comparisons to Monte Carlo (MC) codes, is underway for HZETRN [7]. Several general purpose MC codes are being used for V\&V of HZETRN. These include PHITS, FLUKA, MCNPX, HETCHEDS, MARS, GEANT4 and others [8, 9, 10, 11, 12, 13]. These MC codes are routinely used in $\mathrm{V} \& \mathrm{~V}$ activities of simulated and measured data in space environments, and are particularly important to simulate coupled EM and hadronic cascades. Mesons, leptons and photons are currently not included in the HZETRN transport code [3, 6]. However, most general purpose MC codes include meson, lepton, photon and other secondary particle physics and transport. Each code adopts approximations and simplified treatments to speed up the computation, which can introduce systematic effects. There 
have been numerous investigations that studied the differences in these codes and the implications of embedded assumptions to the final results. A number of investigators who have studied secondary particle production using MC codes, have noted agreements and disagreements in particle fluxes, dose and other related quantities [14, 15, 16, 17, 18, 19]. The differences are attributed to the distinction in the assumptions made in the transport calculations or the differences in the high energy physics models that different codes use. In our literature review, we did not find an effort that quantifies the effects of the meson, lepton and photon transport to space radiation. Some peripheral references were found. For example, Aghara et al. [14, 15] quantified photon production rates from various proton beam energies impinging into Martian and Lunar regolith targets. They noted an increase in the photon production rate at proton kinetic energies $\mathrm{E}>250 \mathrm{MeV}$. They showed that two photons are produced for one proton source particle $(\mathrm{E}>250 \mathrm{MeV})$. This study did not explicitly link the photon production to pions. However, it demonstrated that the rate of photon production increases as the incident energy approaches the pion production threshold energy. Similarly, a number of researchers have compared simulation results with measurements $[20,21,22]$. Most of these studies were geared towards code V\&V and benchmarking. In summary, the literature review reveals that there has not been a conclusive effort to quantify the effects of leptons and mesons and their contribution to absorbed dose in a GCR environment. Hence, calculations performed in this paper are designed specifically to quantify the lepton and meson fluxes and their contribution to the total absorbed dose in a GCR environment. 


\subsection{MCNPX transport}

The MCNPX code package version $2.6 \mathrm{~F}$ is used for all the calculations presented in this paper. It was chosen for three main reasons; the MCNPX code has been used for space radiation research and is well benchmarked, it has an extensive statistical analysis package for tally results, and it provides the user with the ability to selectively turn on and off specific particle physics. We now discuss some pertinent features of MCNPX.

MCNPX was developed at Los Alamos National Laboratory (LANL) and is a three dimensional, general purpose Monte Carlo transport code. It was developed by combining LAHET nuclear physics models and high energy transport to the MCNP coupled neutron, photon, electron code $[10,23]$. MCNP and MCNPX publicly released versions are available through the Radiation Safety Information Computational Center at Oak Ridge National Laboratory (RSICC) and the Organization for Economic Cooperation and Development / Nuclear Energy Agency (OECD/NEA). The last MCNPX general distribution version, version 2.5.0, only transported particles with atomic weight of four and below. The MCNPX code package has been used by a number of investigators to model the complex geometry of spacecraft, and extraterrestrial bases (planetary habitats) in recent years $[14,15,17,22]$. Although these calculations were limited to baryons and light ions up to alpha particles, they provide valuable benchmark verification. The beta version $2.6 \mathrm{~F}$ released in March 2008 added the capability to perform calculations using approximately 2205 heavy ion particles [24]. This new capability in MCNPX enabled a fully coupled, GCR spectral ion simulation.

The MCNPX code package uses the MCNP4C transport package for electron, photon and neutron transport coupled with physics packages for the lepton, baryon, meson and light ion transport. It includes continuous energy transport of 34 different particle types 
(neutron, photon, electron, 5 leptons, 11 baryons, 11 mesons, and 4 light ions) up to $1 \mathrm{TeV}$ per nucleon. The new heavy ion transport and physics is implemented such that users can continue to use the powerful MCNPX capability of modeling a variety of sources (volume, surface, distributed, etc.) and tally options [10, 25]. It also includes an extensive tally analysis package to determine the statistical error in the simulation results. The statistical analysis of the results, a powerful feature in MCNPX, is one of the most critically important requirements for high confidence in MC calculations.

In order to study the effect of pions to the absorbed dose, a code is needed that can turn on and off various physics options without changing the problem setup. MCNPX provides the user with this capability. For example, when the pion channel is turned off, the code still conserves the total energy and charge distribution. It just does not produce pions and transport them. The subsequent pion interactions would not be considered through the phase space. Hence, if the pion physics and transport is not important to a problem, then a statistically significant difference would not surface in the results. In some cases, the secondary particle would not show a significant difference. For example, the secondary particle may not be produced in large numbers or may have a very low interaction probability. In both of these cases, it would lead to a negligible difference in the final results with the channel on and off. It is important that physics and transport changes for a particular particle are imposed without making any changes to the experimental setup (i.e. source, geometry phase space). Any observed difference should be entirely due to the transport and interaction of the particle in the phase space. Lastly, it is important that the physics models are validated against measured data or compared to another code where data is not available. Collot et al. [19] compared pion yield and characteristic spectra between FLUKA and MARS with some measurements. They claim good agreement between data and code simulation results. MCNPX uses the 
same Los Alamos Quark-Gluon String Model (LAQGSM) and Cascade-Exciton Model (CEM) models used in MARS. MCNPX is also well benchmarked for light ions $(\mathrm{A}<4)$, neutron, photons, and electrons. Hence, the addition of the heavy ion source capability makes MCNPX an ideal candidate to investigate the impact of the lepton and meson physics for this study. For the heavy ion transport calculations, MCNPX 2.6F uses the new modified version of the LAQGSM event generator to compute individual interactions with heavy energetic particles and heavy targets [24, 26, 27, 28]. A brief overview of the heavy ion physics implementation in MCNPX is provided in the following section.

\subsection{MCNPX heavy ion physics}

MCNPX utilizes nuclear cross section libraries to transport neutrons, photons, protons and electrons where measured data is available. The measured cross section data is available for some isotopes up to a kinetic energy of approximately $150 \mathrm{MeV}$, with the availability varying based on isotope. The Los Alamos data group updates the cross section data libraries, and once available, these data tables are included into the MCNPX cross section table libraries. For particles and energies where data tables are not available, the code uses physics models [10, 29]. The LAHET code system calculates the generation (spatial and mass distribution) and point of origin of recoiling particles ejected from nuclear reactions, including intranuclear cascades and subsequent fission or evaporation $[30,31]$.

The energy threshold for transition from library and model physics is nuclide dependent, and the transition is handled automatically by a mix-match algorithm in the code. Table 5 shows a condensed description of the transition of event generator codes using mix-match capability. Details of the mix-match capability can be found in the Los Alamos 
report by Hendricks [29]. The user can override these transitions as needed and when possible using physics card options.

Within the hadron model physics region, MCNPX defines two energy regimes based on projectile energy, namely $E \leq 3.5 \mathrm{GeV}$ and $E>3.5 \mathrm{GeV}$. The particle interactions in the energy region between the library physics and $E \leq 3.5 \mathrm{GeV}$ is handled by five different intranuclear cascade (INC) models, which are Bertini, ISABEL, CEM, INCL and LAQGSM. For all of the INC models, the following interaction stages are invoked: nuclear cascade, pre-equilibrium, evaporation or fission, and de-excitation. The user can choose to deactivate specific interactions. For results presented in this paper, the default option was used which invokes all interactions except for the pion off cases. For projectile energies $E \leq 3.5 \mathrm{GeV}$, the hadron physics models of Bertini, FLUKA, CEM03, INCL4 and LAQGSM are used. For heavy ion transport, LAQGSM is used exclusively.

The MCNPX 2.6F physics package includes two significant changes. The recently improved version of the light ion event generator code CEM03, and the full implementation of the LANL version of the LAQGSM, version 3.01 [26, 27, 28]. The CEM03 model describes the nucleon induced reactions at incident energies up to $3.5 \mathrm{GeV}[24,29]$. LAQGSM describes both the particle and nucleus induced reactions at energies above $5 \mathrm{MeV}$ and up to $1 \mathrm{TeV} /$ nucleon for all isotopes. These two new improvements are important to this study. LAQGSM impacts the ability to transport heavy ions, and the improvements in CEM03 impact the light ion and pion transport. A detailed description of the improvements in the LAQGSM and CEM03 event generation codes used in MCNPX 2.6F can be found in Mashnik et al. [27, 28]. For the results described in this paper, the CEM03 model was used exclusively for all the hadrons physics with $E \leq 3.5 \mathrm{GeV}$, and mix-match was used for $E>3.5 \mathrm{GeV}$. The option to force LAQGSM was tried; however, the code showed instability due to errors in handling some specific ion interactions. These 
Table 5: Physics model energy limits for heavy ions, light ions and hadrons.

\begin{tabular}{|c|c|c|c|}
\hline & $\begin{array}{l}\text { Heavy } \\
\text { ions }\end{array}$ & $\begin{array}{l}\text { Light } \\
\text { ions }\end{array}$ & Hadrons \\
\hline \multirow[t]{2}{*}{1} & LAQGSM & ISABEL $(E \leq 1 \mathrm{GeV})$ & Bertini $(E \leq 3.5 \mathrm{GeV})$ \\
\hline & & LAQGSM $(E>1 \mathrm{GeV})$ & FLUKA $(E>3.5 \mathrm{GeV})$ \\
\hline \multirow[t]{2}{*}{2} & LAQGSM & ISABEL $(E \leq 1 \mathrm{GeV})$ & CEM03 $(E \leq 3.5 \mathrm{GeV})$ \\
\hline & & LAQGSM $(E>1 \mathrm{GeV})$ & FLUKA $(E>3.5 \mathrm{GeV})$ \\
\hline \multirow[t]{2}{*}{3} & LAQGSM & INCL4 $(E \leq 1 \mathrm{GeV})$ & $\operatorname{INCL} 4(E \leq 3.5 \mathrm{GeV})$ \\
\hline & & LAQGSM $(E>1 \mathrm{GeV})$ & LAQGSM $(E>3.5 \mathrm{GeV})$ \\
\hline \multirow[t]{2}{*}{4} & LAQGSM & LAQGSM & Bertini $(E \leq 3.5 \mathrm{GeV})$ \\
\hline & & & LAQGSM $(E>3.5 \mathrm{GeV})$ \\
\hline \multirow[t]{2}{*}{4} & LAQGSM & LAQGSM & CEM03 $(E \leq 3.5 \mathrm{GeV})$ \\
\hline & & & LAQGSM $(E>3.5 \mathrm{GeV})$ \\
\hline
\end{tabular}

instabilities were avoided by using the mix-match option. As shown in Table 5, using the mix-match option, either LAQGSM or FLUKA is used for hadron energies $E>3.5 \mathrm{GeV}$. The mix-match algorithm dictates that LAQGSM physics is used first, and if it fails then it is passed to FLUKA. Work is already underway to evaluate the use of LAQGSM exclusively, once these errors in LAQGSM are fixed and available for beta users. However, the exclusive use of LAQGSM is not expected to change the most significant findings detailed in this paper because the GCR spectrum peaks below the transition energy for the LAQGSM physics model $(E>3.5 \mathrm{GeV}$.)

\subsection{Geometry and source}

The main goal of this paper is to quantify the impact of neutral and charged pion production and transport contribution on the downstream dose in human tissue for the 1977 solar minimum GCR spectrum. Results for two different shield thicknesses of Al (5 and $20 \mathrm{~g} / \mathrm{cm}^{2}$ ), followed by $30 \mathrm{~g} / \mathrm{cm}^{2}$ of human tissue, are discussed in this paper. These 
two cases are chosen because these are two standard benchmarks routinely used for $\mathrm{V} \& \mathrm{~V}$ activities. We present particle flux and dose results, and compare the differences in these values with and without pion physics at various depths of $\mathrm{Al}$ and human tissue. The soft tissue elemental composition used was from the International Commission on Radiation Units (ICRU) 33 report[32]. This section describes the details of the MCNPX input.

A simple slab using a combinatorial geometry $(\mathrm{CG})$ description is created for the two benchmark cases of 5 and $20 \mathrm{~g} / \mathrm{cm}^{2}$ of Al shield followed by $30 \mathrm{~g} / \mathrm{cm}^{2}$ of tissue. Both cases are modeled individually to avoid any particle backscatter contributions and also to verify the results separately. The GCR source is modeled as a planar source uniformly distributed on a surface. It is modeled as an isotropic source, directionally biased towards the shield. Figure 1 shows the simulated geometry with the $\mathrm{Al}$ and tissue thickness, and a wire frame GCR source plane centered on the left hand side. The dose segments are shown as solid colors, and the $\mathrm{Al}$ and tissue slab is shown as transparent solids.

The source is modeled explicitly with 26 heavy ions, helium (alpha) and hydrogen (proton) ions, with the maximum kinetic energy of $5 \mathrm{GeV}$ per nucleon. The source sampling rate is determined by the relative abundance of each element in the 1977 solar minimum GCR event. The GCR source boundary condition is calculated using the 2004 Badhwar-O'Niell GCR model referred to as the B-O'04 Model [33]. Figure 2 shows the ion abundance curve for the 1977 solar minimum GCR event as predicted by the B-O'04 model. Figure 3 shows the differential flux of the nine ions for the 1977 solar minimum GCR spectrum. 


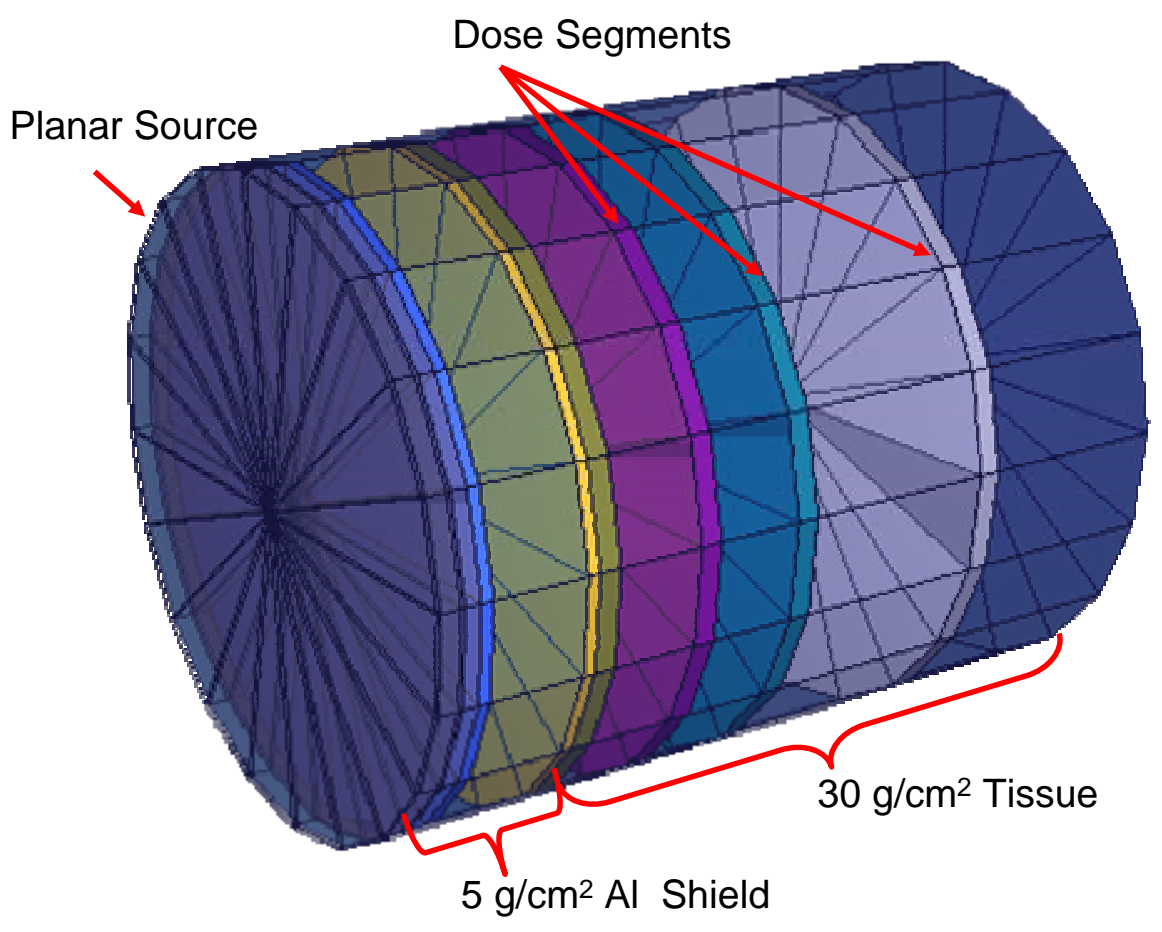

Figure 1: Plot of MCNPX geometry.

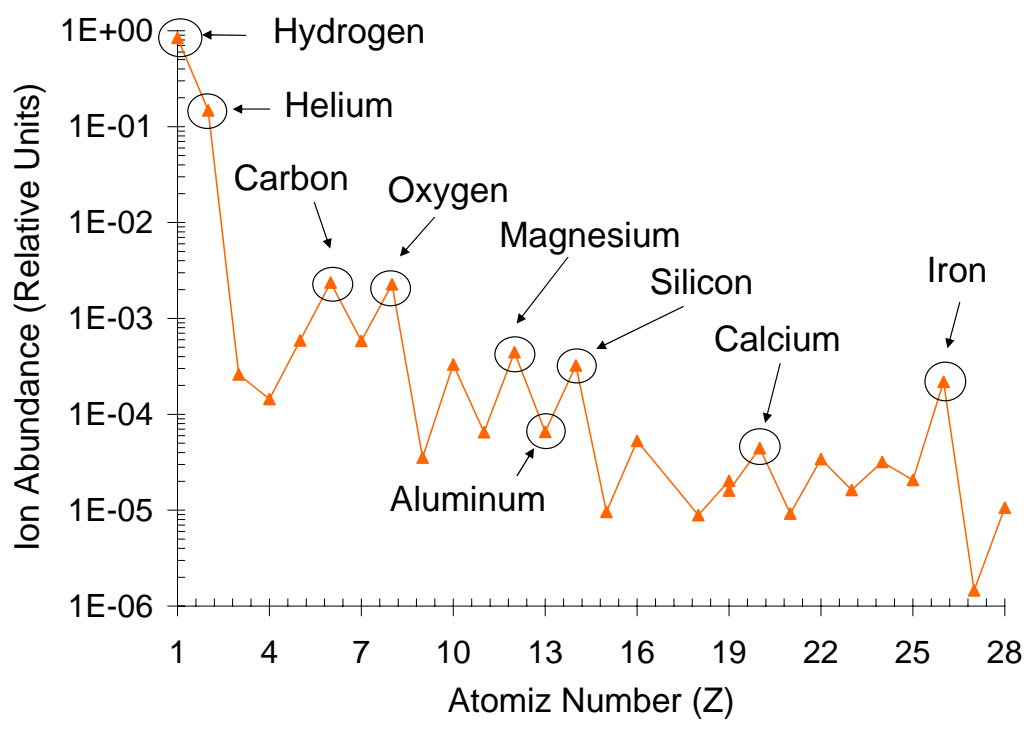

Figure 2: Galactic cosmic ray (GCR) ion abundance. 


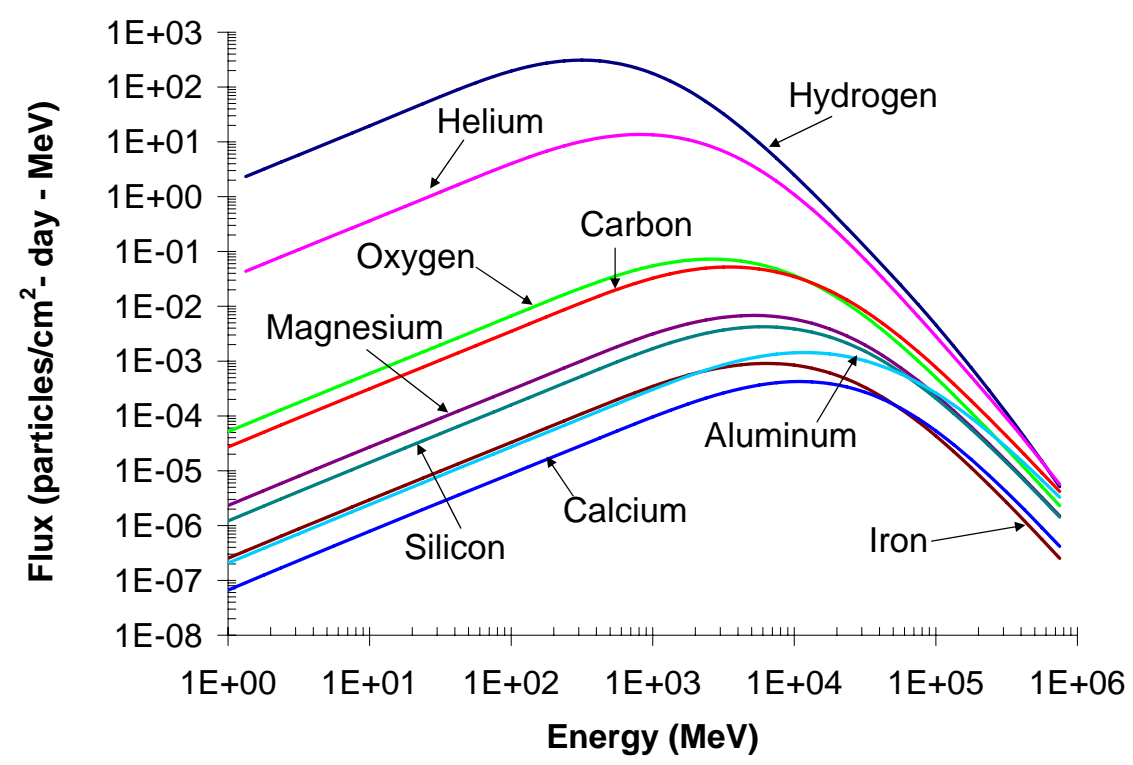

Figure 3: Differential flux of 1977 GCR ions.

\subsection{Tallies}

MCNPX offers seven standard current and flux tally options. These, in combination with tally modifiers and multipliers, can be used to create a large selection of tally options. For the purpose of this analysis, a combination of four different tallies were used: surface current tally, flux tally, energy deposited per track length tally and collision heating tally. The cosine and energy multiplier cards and the "res" card were used to generate the results from MCNPX. The energy deposited per unit weight values were tallied at various $\mathrm{Al}$ and tissue depths. These values are related to absorbed dose in units of cGy. The following conversion factor is used: $1 \mathrm{MeV}$ per gram is equal to $1.6 \times 10^{-8} \mathrm{cGy}$. For convenience, the energy per gram will be referred to as dose. 


\section{RESULTS AND DISCUSSION}

All calculations presented in this paper are performed with MCNPX version 2.6F. For hadron transport $E \leq 3.5 \mathrm{GeV}$, the CEM03 model is used exclusively; for kinetic energies $E>3.5 \mathrm{GeV}$, the mix-match option is used. For light ions, the mix-match physics option is used. The heavy ion transport is performed with the LAQGSM nuclear cascade/evaporation model. The calculations are performed to meet a minimum of $10 \%$ or less statistical uncertainty in the evaluated data. For all results, the error bars are plotted on the curves. In most cases, they are not visible because the relative error is very small. The calculations are performed on a 60-node (120 CPU) cluster, "Poe", at NASA Langley Research Center. "Poe" is a LINUX cluster, with an AMD Dual core 2.4 GHz Opteron chip set with 2 GB dedicated memory per core. The MCNPX code version 2.6F is built with Intel 10.1 FORTRAN and a $\mathrm{C}++$ compiler on a Fedora Core 4 Linux operating system.

Two broad categories of results are presented in this paper, which are the flux and the dose results. For flux results, in most cases, the results are per source particle, rather than absolute values. Light ions $(A \leq 4)$ include deuterons, tritons, helions and alphas. Heavy ions $(A>4)$ include all possible ions (2205 isotopes) heavier than alphas. Comparisons are made between flux and dose results for the pion channel turned on and off. The pion transport includes the charged and neutral pion channels. For all calculations, the neutron, photon, electron, light ion and heavy ion channels are included. The only difference between the pion on and pion off results is the presence or absence of the pion channel.

A set of standard checks was performed to verify that the source and geometry were modeled correctly. For example, primary particle flux and absorbed dose values 
at different thicknesses in $\mathrm{Al}$ and tissue were compared to standard benchmark results from HZETRN. These checks are important to assure that differences observed in the final results are truly due to pion physics. In this paper, we discuss the total flux versus depth curves for neutrons, photons, and electrons. Next, the particle differential fluxes for neutrons and photos are presented. Finally, the dose results are presented. The dose results include dose-depth curves and relative dose contributions from various secondary products to the total dose.

\subsection{Flux Results}

Figures 4 and 5 show the total particle flux versus depth curves for neutrons, photons, and electrons in $\mathrm{Al}$ and tissue for the case with pion channel on and off. We note the build up of neutrons, photons, and electrons fluxes as a function of depth. Neutron flux in $\mathrm{Al}$ changes gradually due to the presence of pion channel. The percent change in neutron flux starts to be above $5 \%$ at $\mathrm{Al}$ depths of $10 \mathrm{~g} / \mathrm{cm}^{2}$ and greater. In tissue, the difference in neutron flux between pion channel on and off is more easily visible. This is because the neutrons are produced from charged pion and photon interactions.

The most interesting result from these plots is the difference in the rate of buildup of photon and electron fluxes in $\mathrm{Al}$ when compared between the pion on and pion off case. Notice that for the pion off case, the photon curve parallels the neutron curve, as most photons are produced from nuclear interactions. However, in the pion on case, the photon curve is much higher than the neutron flux. The increase in photons flux is due to the contributions from neutral pion decay, EM cascades and the charged pion interactions. These results relate to the theoretical discussion on mean lifetime and range of pions, as shown in Table 3. Note that neutral pions have very short range and immediately 


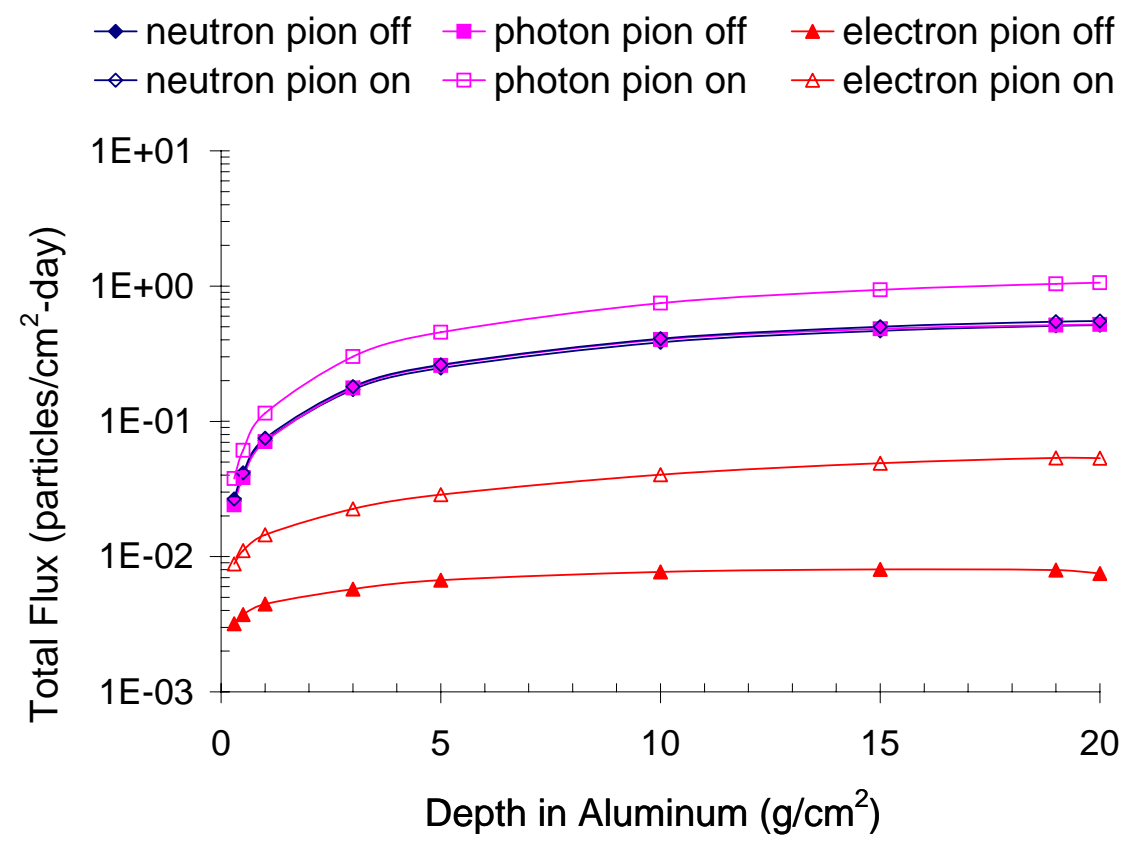

Figure 4: Neutron, photon, electron flux at various depths of $\mathrm{Al}$ with pion channel on and off.

decay into photons. The charged pions, once produced, travel deeper and undergo nuclear interactions. We notice in figures 4 and 5 that photon and charged electron fluxes increase as a function of depth. The photon production from different interactions is clear when we examine the differential flux for neutrons and photons.

Figures 6 and 7 show the neutron and photon differential spectra for the pion channel on case. The neutron and photon plots are shown at the 5 and $20 \mathrm{~g} / \mathrm{cm}^{2} \mathrm{Al}$ depths. Additionally, neutron and photon spectra at $30 \mathrm{~g} / \mathrm{cm}^{2}$ tissue depth behind $20 \mathrm{~g} / \mathrm{cm}^{2} \mathrm{Al}$ are shown in the same plots. Notice that the neutron and photon spectral intensity is greater as the primary source particles pass through Al. Neutron and photon intensity for the $20 \mathrm{~g} / \mathrm{cm}^{2}$ case is greater than the $5 \mathrm{~g} / \mathrm{cm}^{2}$ case.

A close examination of figure 7 reveals that there are three characteristic production regions in the photon spectra. The higher energy region $(E \geq 50 \mathrm{MeV})$, with peak at $\sim$ 


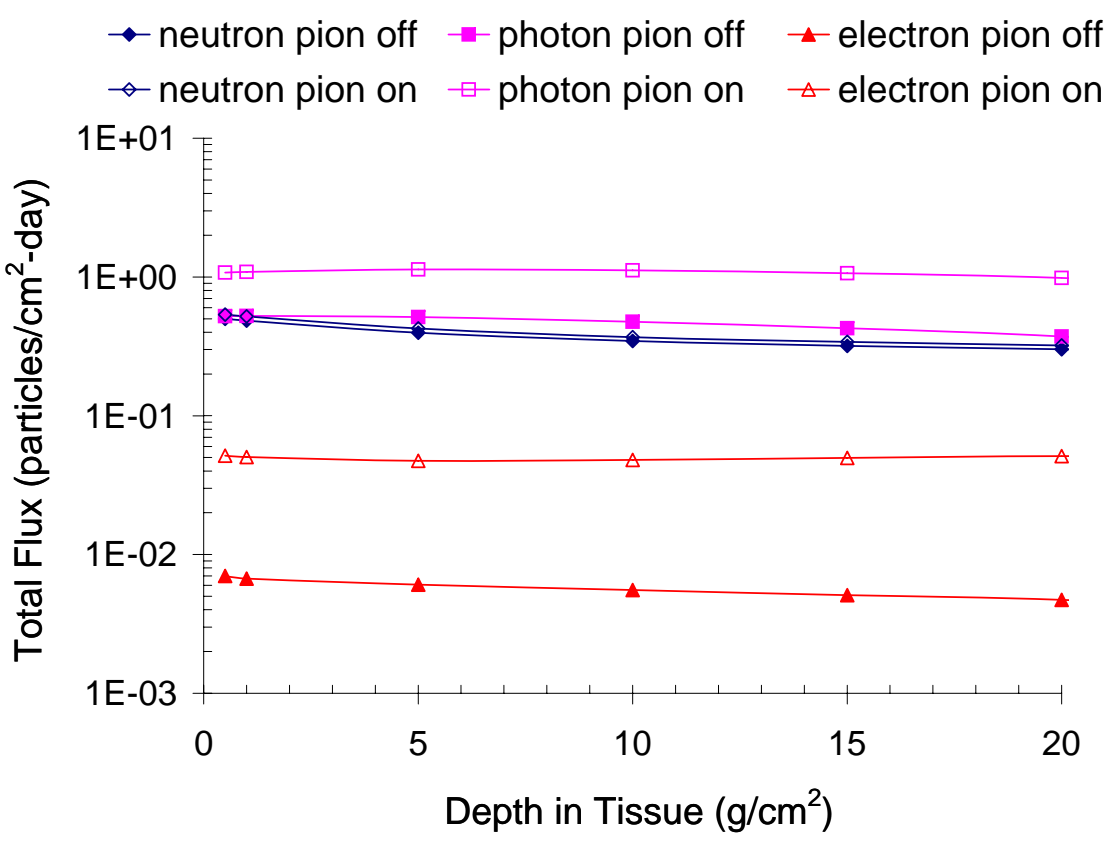

Figure 5: Neutron, photon, electron at various depths of tissue behind $20 \mathrm{~g} / \mathrm{cm}^{2}$ of $\mathrm{Al}$ with pion channel on and off.

$100 \mathrm{MeV}$, is exclusively from neutral pion decay. The intermediate region $(1 \mathrm{MeV}-50$ $\mathrm{MeV}$ ) is from nuclear interactions including neutron scattering reactions. Lastly, the low energy region $(\mathrm{E}<1 \mathrm{MeV})$ is mainly from decay gammas and low energy Bremsstrahlung. Clearly, the pion produced photons, being energetic, would travel to significant depth. Consequently, these photons deliver radiation dose at greater depths. This is evident when we compare the photon spectra at 5 and $20 \mathrm{~g} / \mathrm{cm}^{2}$ of $\mathrm{Al}$ depths. The photon spectra remain practically unaltered at higher energies $(E \geq 100 \mathrm{MeV})$, and the photon flux increases in the intermediate energy range. Figure 8 shows clearly the difference in the photon flux due to the presence of pions. It is evident that by transporting pions the high energy photons component is present.

Finally, let us examine the relative attenuation of neutrons and photons as they travel through $30 \mathrm{~g} / \mathrm{cm}^{2}$ tissue. Once in tissue, note that the lower energy neutrons $(\mathrm{E}<50$ 


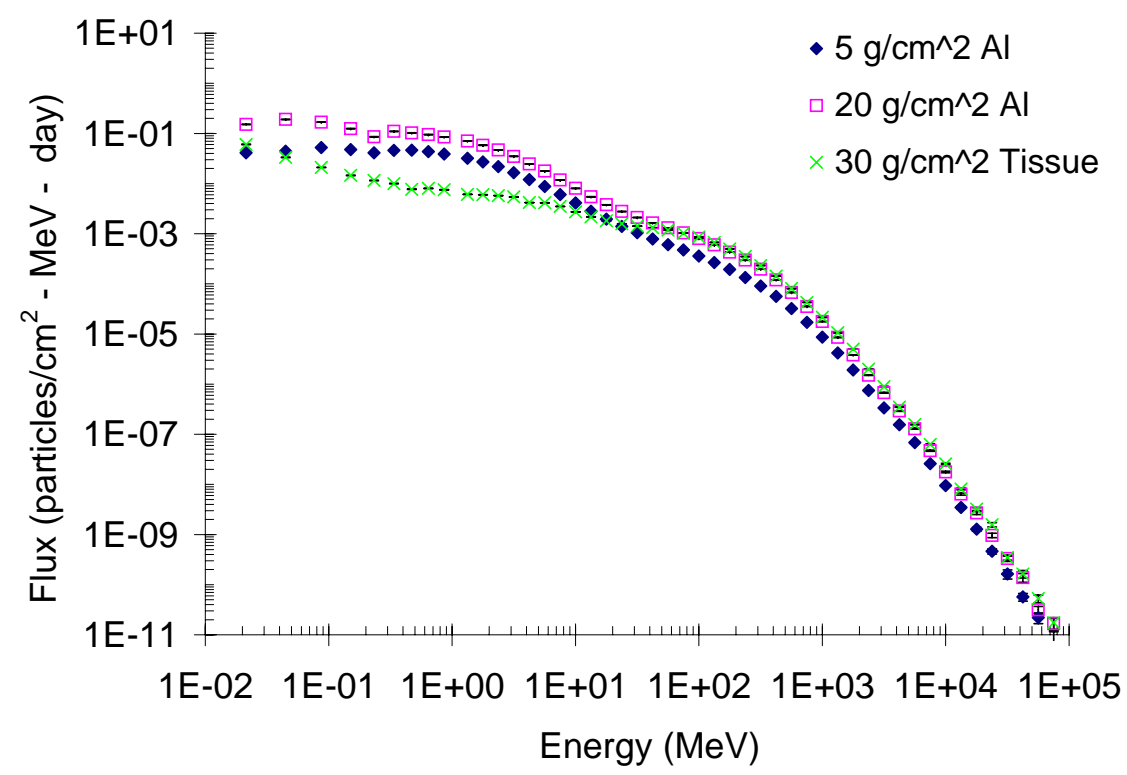

Figure 6: Neutron flux behind $5 \mathrm{~g} / \mathrm{cm}^{2}, 20 \mathrm{~g} / \mathrm{cm}^{2} \mathrm{Al}$ and at $30 \mathrm{~g} / \mathrm{cm}^{2}$ tissue.

$\mathrm{MeV}$ ) attenuate. However, the high energy neutrons remain unaltered. Also, note that the $1 \mathrm{MeV}$ neutron flux is reduced by an order of magnitude. However, in the case of photons, we notice that there is in fact a slight increase in high energy photons and only a small reduction in the lower energy photons. This significantly changes the make up of the secondary radiation products and their delivered dose as these photons travel through spacecraft and human tissue. Further characterization of the photon spectrum to evaluate its effect on dose equivalent is already underway.

Tables 6 and 7 show the percent change in total particle flux due to the presence of pions in $\mathrm{Al}$ and tissue, respectively. It is clear from these tables that by including the pion transport, the particle flux for electrons and photons increases considerably both in $\mathrm{Al}$ and in tissue. More importantly, the total flux for neutrons changes steeply, with values of about $7 \%$ at $20 \mathrm{~g} / \mathrm{cm}^{2}$ in $\mathrm{Al}$, as seen in Table 6 . The difference remains relatively flat at about $6.5 \%$ in tissue. (See Table 7). Similarly, the light ion flux increases sharply (about 3\%) in $\mathrm{Al}$ and then remains flat throughout the depth of tissue. The proton flux 


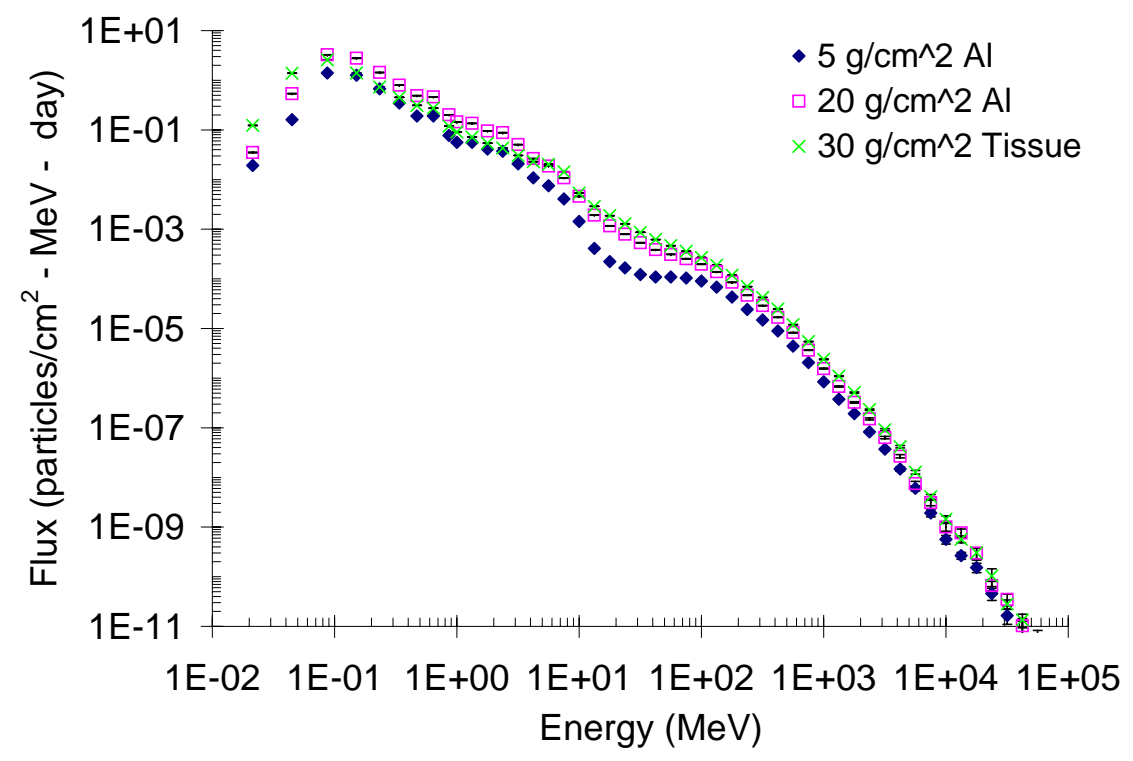

Figure 7: Photon flux behind $5 \mathrm{~g} / \mathrm{cm}^{2}, 20 \mathrm{~g} / \mathrm{cm}^{2} \mathrm{Al}$ and at $30 \mathrm{~g} / \mathrm{cm}^{2}$ tissue.

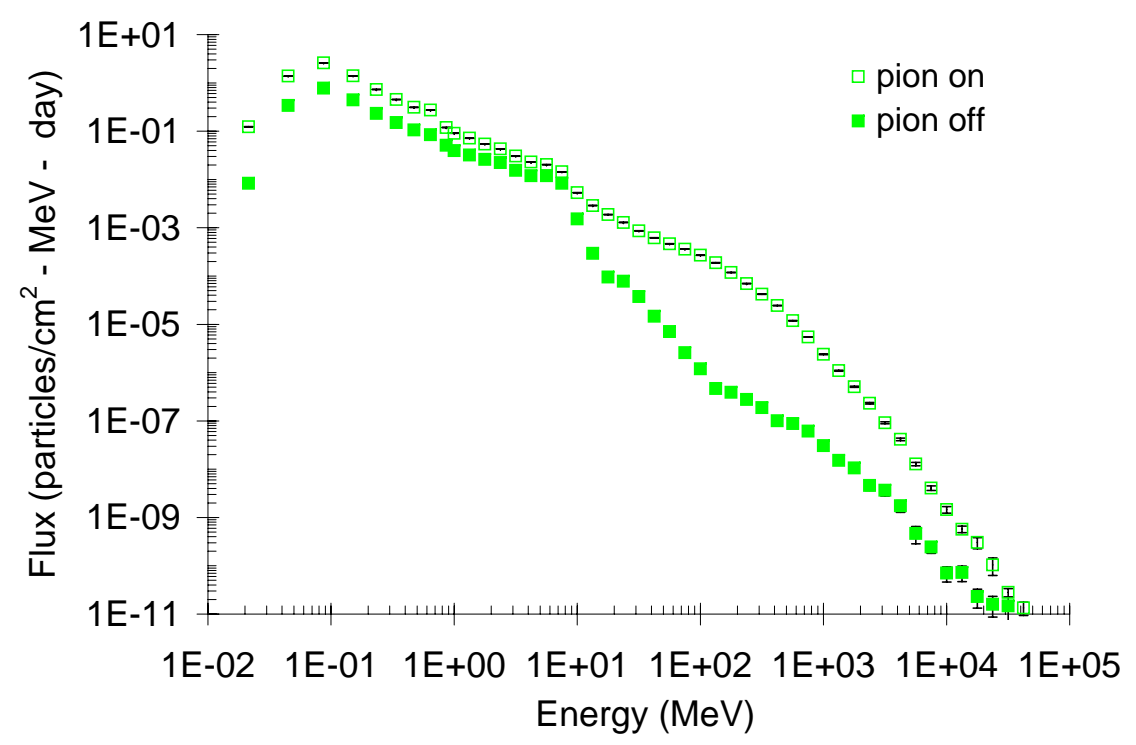

Figure 8: Photon flux behind at $30 \mathrm{~g} / \mathrm{cm}^{2}$ tissue behind $20 \mathrm{~g} / \mathrm{cm}^{2} \mathrm{Al}$. 
Table 6: Percent difference in total flux at various $\mathrm{Al}$ depths.

\begin{tabular}{ccccccc}
\hline $\begin{array}{c}\text { Depth in } \\
\text { Aluminum } \\
\text { [cm] }\end{array}$ & Electrons & Photons & Neutrons & lons $(\mathrm{A} \leq 4)$ & Protons & Ions $(\mathrm{A}>4)$ \\
\hline 1.0 & $69.2 \%$ & $38.6 \%$ & $3.9 \%$ & $1.9 \%$ & $0.0 \%$ & $0.0 \%$ \\
3.0 & $74.5 \%$ & $41.4 \%$ & $4.7 \%$ & $2.3 \%$ & $0.1 \%$ & $0.0 \%$ \\
5.0 & $76.6 \%$ & $43.0 \%$ & $5.2 \%$ & $2.4 \%$ & $0.1 \%$ & $0.0 \%$ \\
10.0 & $80.9 \%$ & $46.1 \%$ & $6.1 \%$ & $2.3 \%$ & $0.2 \%$ & $0.0 \%$ \\
15.0 & $83.6 \%$ & $48.6 \%$ & $6.6 \%$ & $2.8 \%$ & $0.3 \%$ & $0.0 \%$ \\
20.0 & $86.0 \%$ & $51.0 \%$ & $7.0 \%$ & $2.7 \%$ & $0.4 \%$ & $-0.1 \%$ \\
\hline
\end{tabular}

Table 7: Percent difference in total flux at various tissue depths.

\begin{tabular}{ccccccc}
\hline $\begin{array}{c}\text { Depth in } \\
\begin{array}{c}\text { Tissue } \\
{[\mathrm{cm}]}\end{array}\end{array}$ & Electrons & Photons & Neutrons & lons $(\mathrm{A} \leq 4)$ & Protons & lons $(\mathrm{A}>4)$ \\
\hline 1.0 & $86.7 \%$ & $52.0 \%$ & $6.9 \%$ & $2.4 \%$ & $0.4 \%$ & $0.1 \%$ \\
5.0 & $87.2 \%$ & $54.7 \%$ & $6.6 \%$ & $2.2 \%$ & $0.5 \%$ & $0.1 \%$ \\
10.0 & $88.5 \%$ & $57.4 \%$ & $6.3 \%$ & $2.6 \%$ & $0.6 \%$ & $0.1 \%$ \\
15.0 & $89.7 \%$ & $59.8 \%$ & $6.3 \%$ & $2.3 \%$ & $0.7 \%$ & $0.3 \%$ \\
20.0 & $90.8 \%$ & $62.1 \%$ & $6.3 \%$ & $3.1 \%$ & $0.8 \%$ & $0.4 \%$ \\
30.0 & $93.1 \%$ & $66.5 \%$ & $6.2 \%$ & $2.7 \%$ & $1.0 \%$ & $1.1 \%$ \\
\hline
\end{tabular}

increases slightly in both $\mathrm{Al}$ and in tissue. The heavy ion flux remains largely unaltered in $\mathrm{Al}$ and in tissue when compared for the pion on and off cases. The change in total flux results are consistent with the theoretical discussion of the previous section. Due to pion interactions, we expect and notice an increase in particle flux for neutron, light ion and the electromagnetic particles (photons and electrons). Further study on kaons and muons is underway.

\subsection{Dose Results}

Figure 9 shows the relative dose contribution of various radiation constituents to the total dose at $30 \mathrm{~g} / \mathrm{cm}^{2}$ of tissue after the primary radiation has passed through $5 \mathrm{~g} / \mathrm{cm}^{2} \mathrm{Al}$ shielding for pions off and pions on, respectively. In the previous section, we noticed a 
change in flux due to the presence of pion physics. Let us now examine the effect on dose. Comparing the charts in figure 9 , we notice a significant difference in photon contribution to dose. It changes from about $1 \%$ to $9 \%$ for the pion off to pion on case, respectively; an increase by a factor of $9(89 \%)$. The increase in dose is related to the high energy photons $(\mathrm{E}>100 \mathrm{MeV})$ that are produced from the pion channel. The direct pion contribution (through charged pion interactions) is about $3 \%$. The total dose contribution of pions and photons combined is about $13.5 \%$. We do not see much change in neutron and heavy ion contributions to the total dose. The dose contribution from light ions decreases by about $20 \%$. For protons, the dose contribution decreases by about $10 \%$. We note a change in makeup of the relative dose contributions to total dose from individual constituents. This is a significant and important finding. The implication of this change to dose equivalent are being investigated.

Figure 10 shows the results for the $20 \mathrm{~g} / \mathrm{cm}^{2}$ of $\mathrm{Al}$. The change in dose trends for the $20 \mathrm{~g} / \mathrm{cm}^{2}$ of $\mathrm{Al}$ case are similar to the $5 \mathrm{~g} / \mathrm{cm}^{2}$ case. The changes are even greater in the $20 \mathrm{~g} / \mathrm{cm}^{2}$ of $\mathrm{Al}$ case. The photon dose contribution changes to $13 \%$ (pion on) from $1 \%$ (pion off). Hence, the photon dose increases by about $92 \%$. The direct pion dose contribution is about $5 \%$. The total combined contribution for photons and pions of about $18 \%$, is significant. The heavy ion dose contribution remains the same between the pion on and off cases. The overall heavy ion dose contribution decreases from $3 \%$ to $2 \%$ when compared to the $5 \mathrm{~g} / \mathrm{cm}^{2}$ of $\mathrm{Al}$ case. This is expected, as additional $\mathrm{Al}$ shielding breaks up and attenuates the primary heavy ions, resulting in a lower direct dose contribution from heavy ions at deeper depths. The relative dose contribution of neutrons to total dose changes from $5 \%$ to $4 \%$ (20\% decrease). Similarly, the light ion dose contributions changes from $13 \%$ to $11 \%$ ( $15 \%$ decrease); whereas, the proton dose contributions changes from $79 \%$ to $65 \%$ (18\% decrease). 

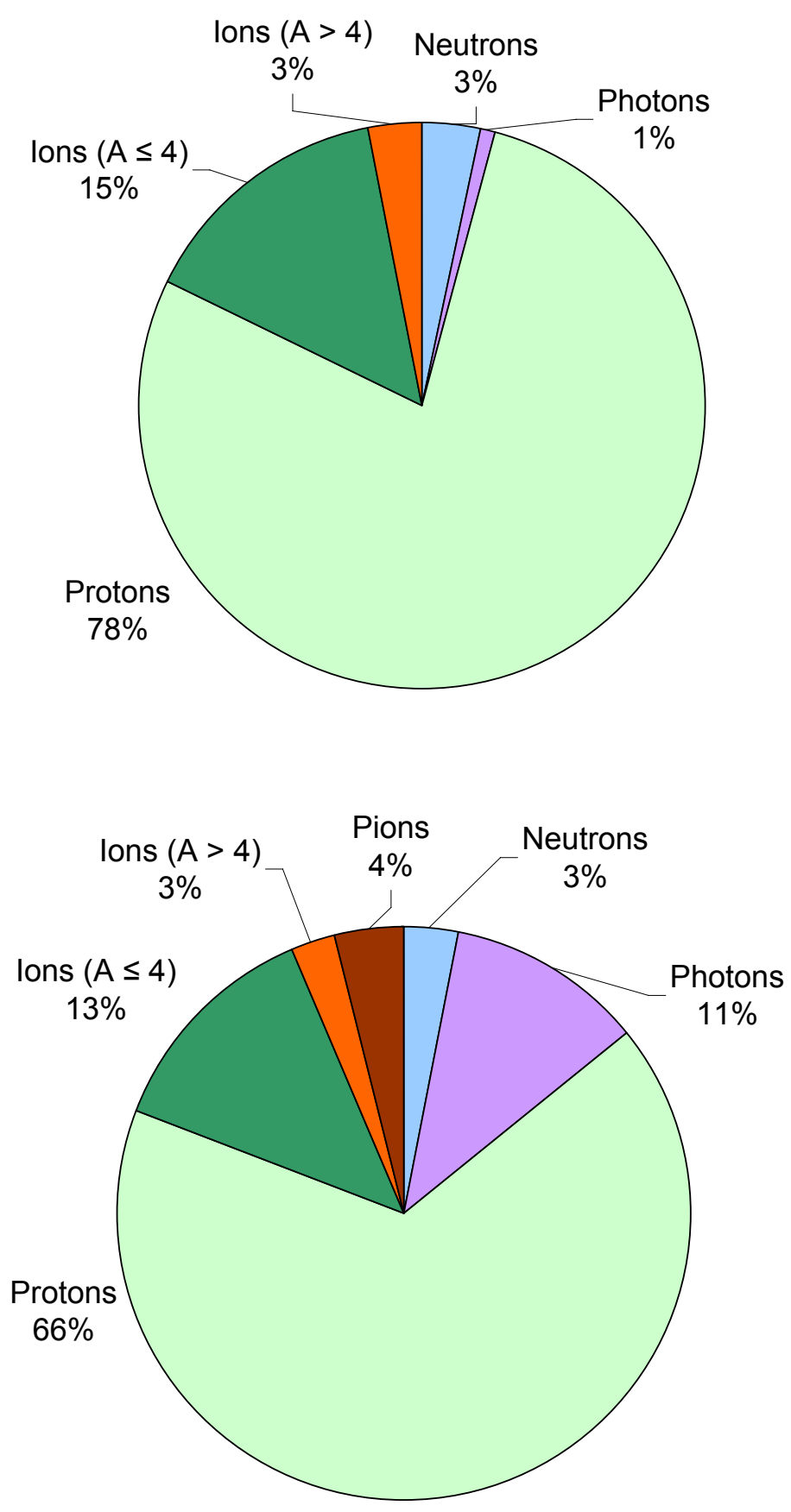

Figure 9: Relative contribution of various constituents to total dose behind $5 \mathrm{~g} / \mathrm{cm}^{2}$ of $\mathrm{Al}$ and $30 \mathrm{~g} / \mathrm{cm}^{2}$ of tissue, with the pion channel off (upper figure) and on (lower figure). 
It is important to point out that the changes noted in the dose contributions are in the relative contribution of each constituent to the total dose. As we will see later, the total dose increases due to the pion channel. Additionally, the dose from each of the constituents increases deeper into the tissue. Figures 9 - 10 showed us how the relative contributions change as the pion physics and transport are included.

Figures 11 - 12 show the relative dose contribution to total dose results for the $5 \mathrm{~g} / \mathrm{cm}^{2}$ and $20 \mathrm{~g} / \mathrm{cm}^{2}$ of $\mathrm{Al}$ at various tissue depths. These results show that there is clearly a significant change in the makeup of dose contributions to total dose from individual constituents due to the presence of the pion channels.

Figure 13 shows the percent change in dose at various tissue depths for each individual constituent due to the presence of the pion channels. The results shown are for the $20 \mathrm{~g} / \mathrm{cm}^{2}$ of $\mathrm{Al}$. The trends for the $5 \mathrm{~g} / \mathrm{cm}^{2}$ of $\mathrm{Al}$ case are very similar and hence not shown here. Notice that the dose from light and heavy ions increases by about $5 \%$ at $30 \mathrm{~g} / \mathrm{cm}^{2}$ of tissue depth. The light ion dose increases by about $2 \%$ at the surface of the tissue $\left(0 \mathrm{~g} / \mathrm{cm}^{2}\right)$, with a gradual increase as a function of tissue depth. For heavy ions, the slope is greater from $0 \mathrm{~g} / \mathrm{cm}^{2}$ to $30 \mathrm{~g} / \mathrm{cm}^{2}$ tissue depth. The neutron dose changes by nearly $9 \%$, which is a noticeable increase. The slope for the neutron dose remains flat through the tissue depth, consistent with the neutron flux results. It reaches maximum at approximately middle depth in tissue. The contribution from protons changes by a smaller amount of about $2.5 \%$. This relatively smaller change in proton dose can be explained. We note that the dose from protons is dominated by the primary source particles. Hence, the relative increase in proton dose due to the pion channel does not show a significant change, as it does for the neutrons. An important finding from these results is the overall increase in dose from hadrons and ions deeper into the tissue depth. This is significant because by ignoring pions and EM particles, the dose contributions to critical organs 

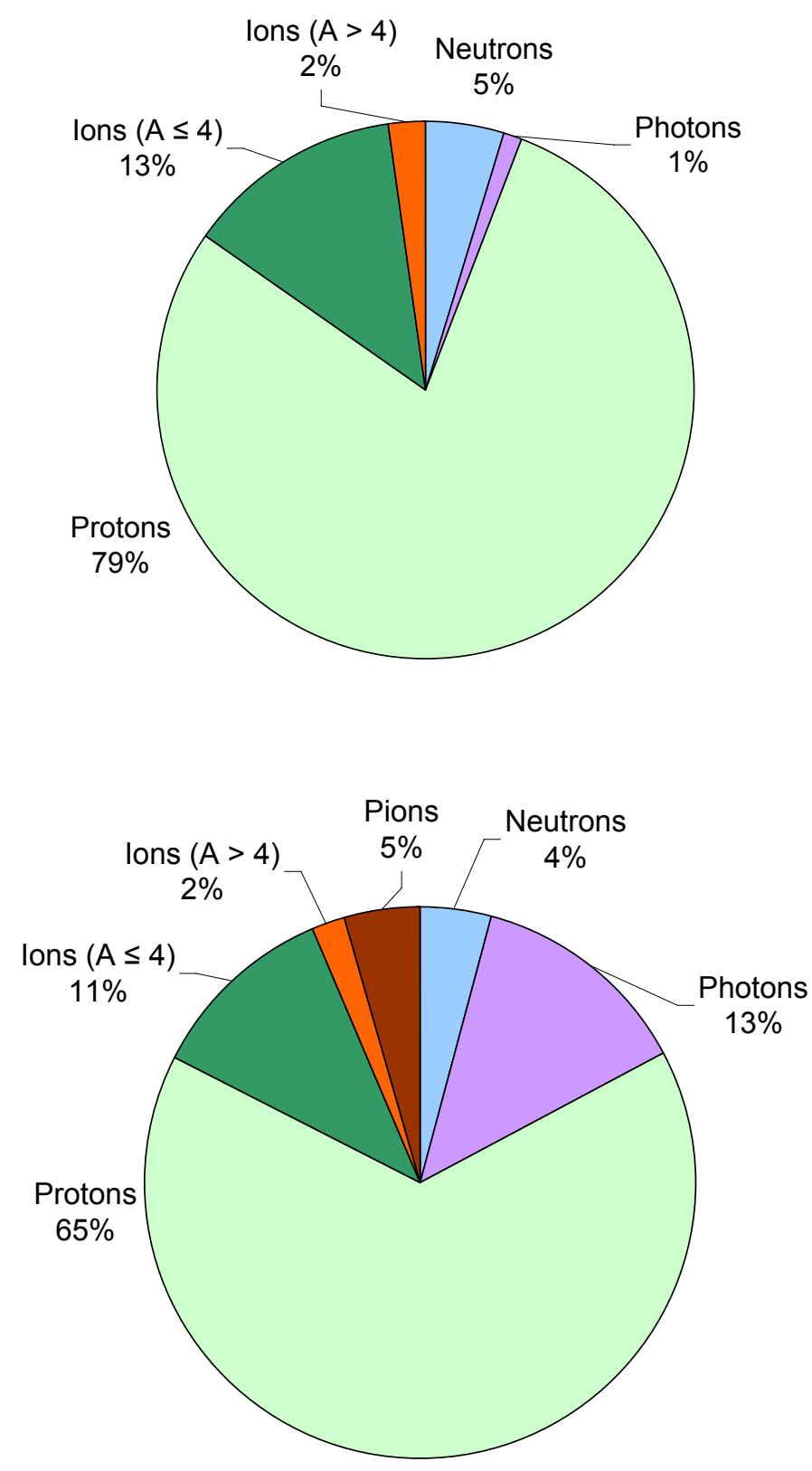

Figure 10: Relative contribution of various constituents to total dose behind $20 \mathrm{~g} / \mathrm{cm}^{2}$ of $\mathrm{Al}$ and $30 \mathrm{~g} / \mathrm{cm}^{2}$ of tissue, with the pion channel off (upper figure) and pion channel on (lower figure). 

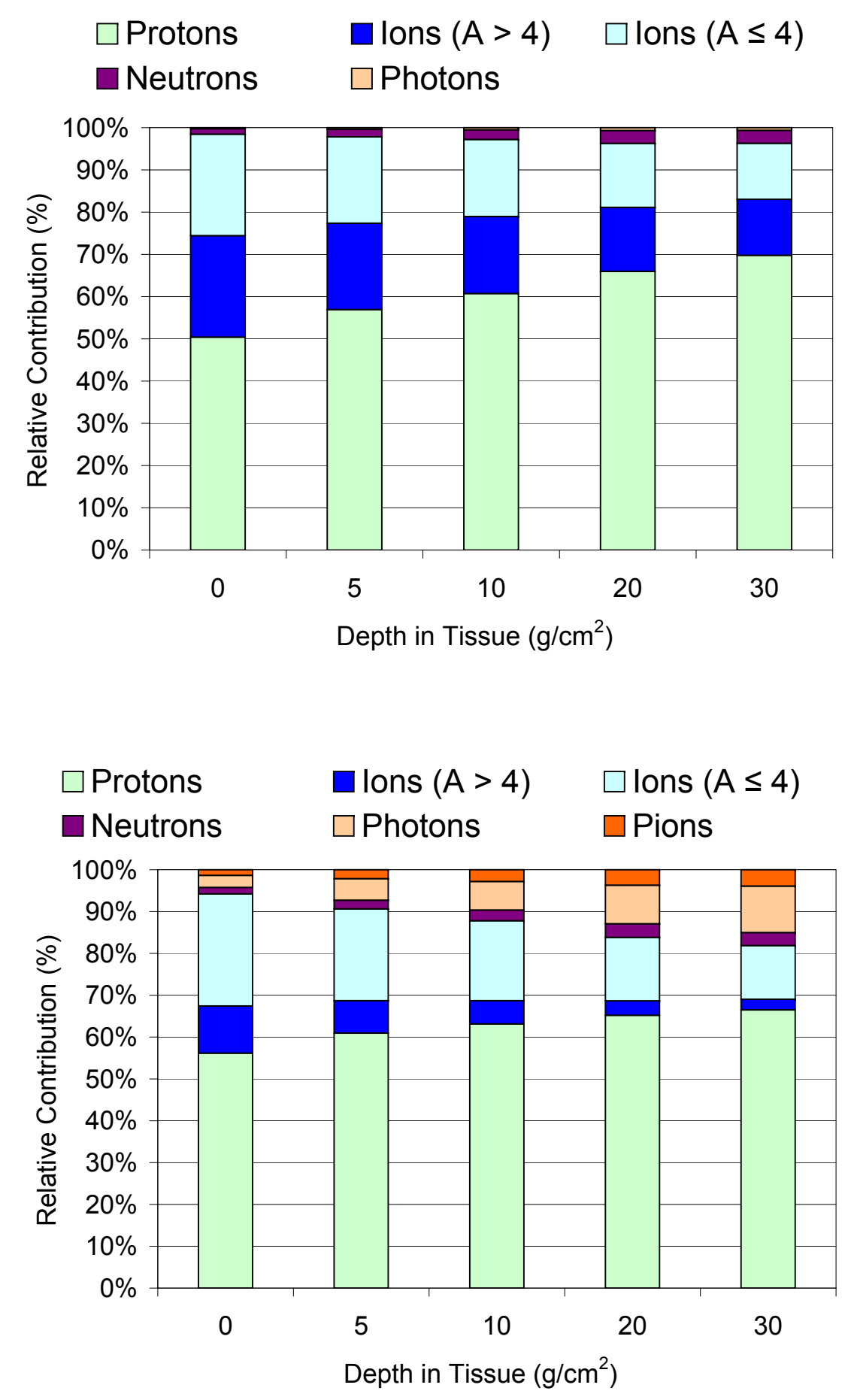

Figure 11: Relative contribution of various constituents to total dose behind $5 \mathrm{~g} / \mathrm{cm}^{2}$ of $\mathrm{Al}$, with the pion channel off (upper figure) and pion channel on (lower figure). 

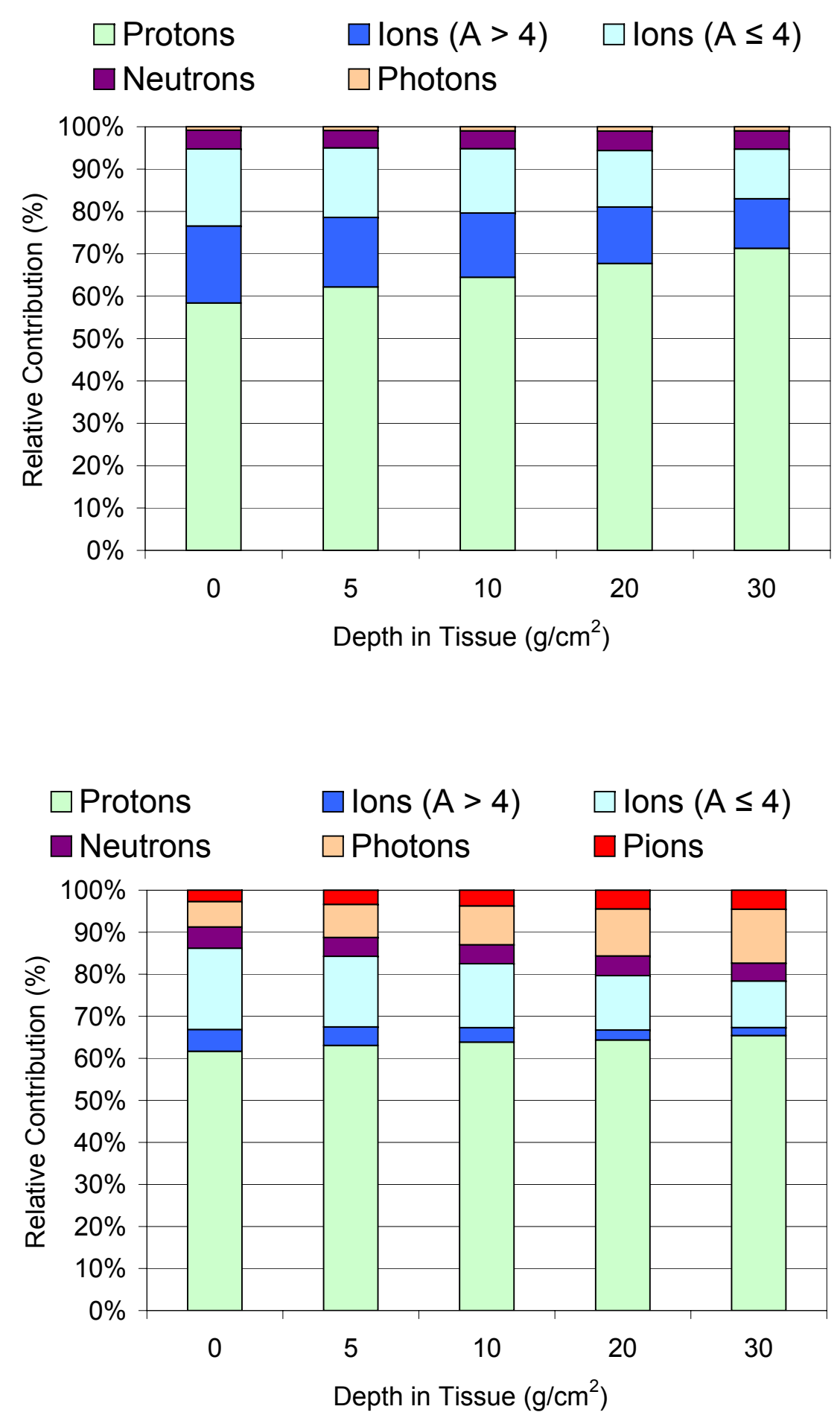

Figure 12: Relative contribution of various constituents to total dose behind $20 \mathrm{~g} / \mathrm{cm}^{2}$ of $\mathrm{Al}$, with the pion channel off (upper figure) and pion channel on (lower figure). 


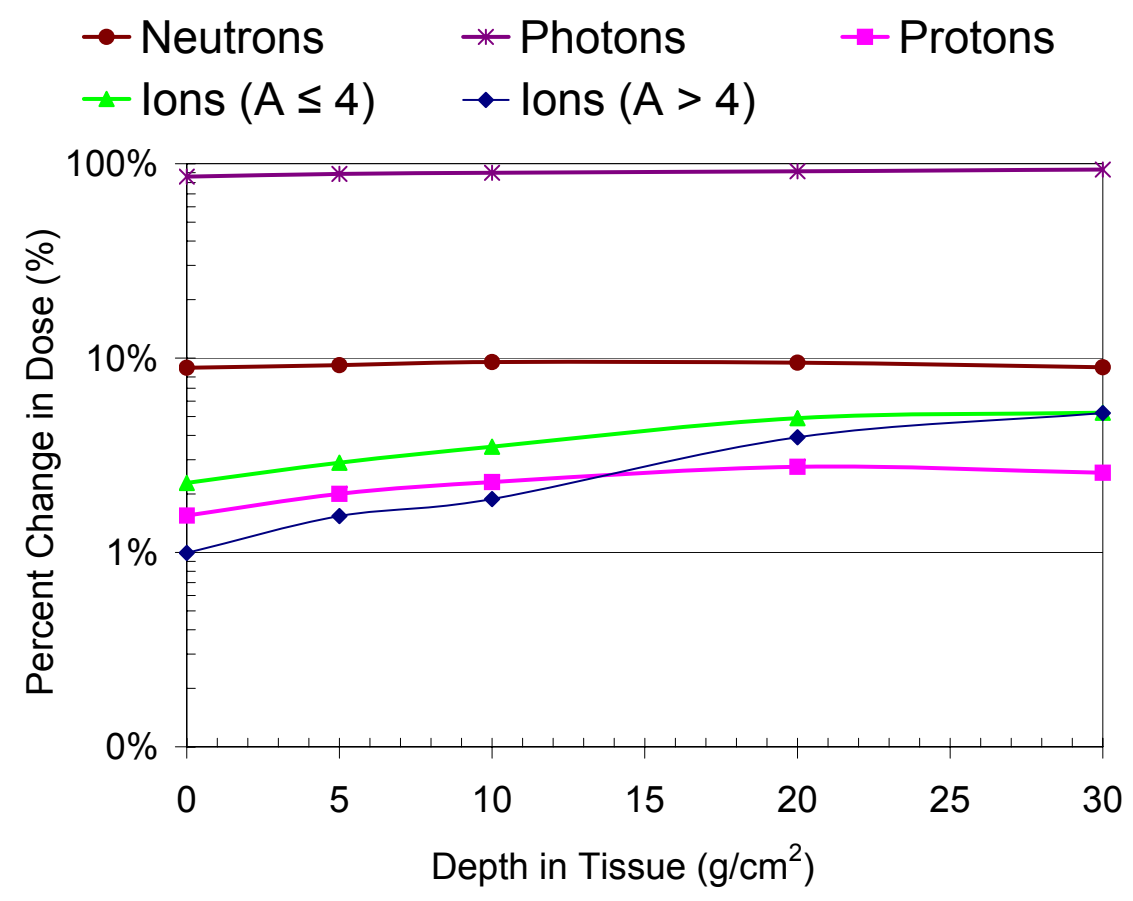

Figure 13: Percent increase in dose deposited by radiation constituents at various depths of tissue behind $20 \mathrm{~g} / \mathrm{cm}^{2}$ of $\mathrm{Al}$.

could be under predicted. This could lead to misinterpretation of shielding effectiveness. Further investigation using linear energy transfer (LET) and dose equivalent is needed to fully evaluate the broader impact of these particles.

Figure 14 shows the increase in total dose as a function of tissue depth behind $5 \mathrm{~g} / \mathrm{cm}^{2}$ and $20 \mathrm{~g} / \mathrm{cm}^{2} \mathrm{Al}$. We observe that the increase in total dose is nearly $16 \%$ due to the presence of the pion when the primary GCR spectrum travels through $20 \mathrm{~g} / \mathrm{cm}^{2}$ of $\mathrm{Al}$ and $30 \mathrm{~g} / \mathrm{cm}^{2}$ of tissue. For the $5 \mathrm{~g} / \mathrm{cm}^{2}$ case, the total dose increases by about $9 \%$. In summary, we notice a net increase in total dose when the pion channel is turned on. We observe that this increase is attributed to both increased nuclear interactions and EM cascades. An increase in dose as a function of tissue depth from all constituents is also noted. 


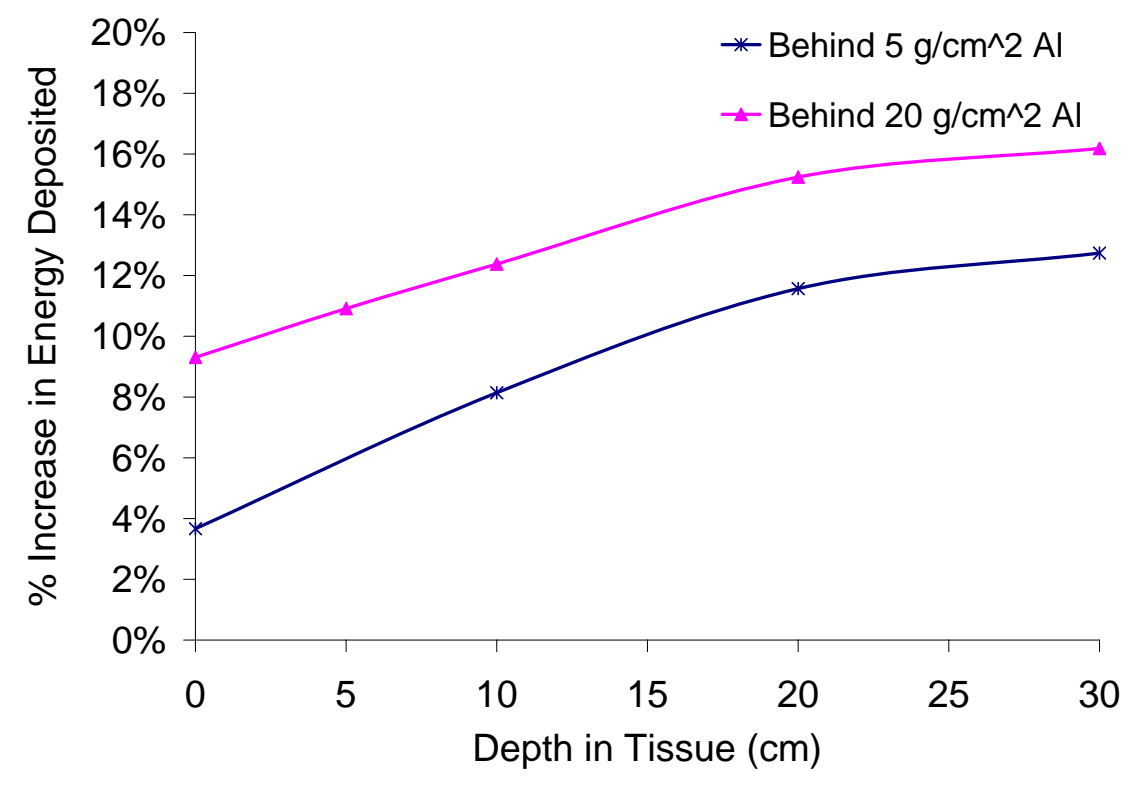

Figure 14: Percent increase in dose deposited at various depths of tissue behind 5 and $20 \mathrm{~g} / \mathrm{cm}^{2}$ of Al.

\section{CONCLUSIONS}

Results of this study suggest that the inclusion of meson, lepton and photon physics for space radiation shielding calculations may be necessary. Further investigation of this effect on dose equivalent and linear energy transfer (LET) is being pursued to quantify the effects of mesons on integral quantities. Both the flux and dose results suggest that inclusion of these particles will affect the overall dose prediction and the flux distribution of hadrons. This study shows a statistically significant difference in the contribution of absorbed dose from the various secondary constituents to the total absorbed dose when pion transport is included, as substantial increase in photon flux and dose is observed. The photon spectrum shows three clear regions of particle production. A considerable portion of the spectrum is at energies greater than $100 \mathrm{MeV}$, which is a potential concern for shielding consideration. HZETRN has proved to be an efficient and a reliable tool 
for space radiation shielding evaluation for NASA. Since the underlying physics and transport are handled differently in HZETRN and MC codes, specific code comparison benchmarks must be developed to investigate the impact of these findings. Currently, $\mathrm{V} \& \mathrm{~V}$ efforts are underway to compare these results with other MC codes. These results suggest that the $\mathrm{V} \& \mathrm{~V}$ efforts of the transport codes for space radiation must further evaluate the meson contribution to human and electronics exposure inside spacecraft.

Acknowledgements: We thank Dr. Ryan Norman for reviewing the manuscript. This research was supported by NASA Administrator's Fellow Program (NAFP) managed by United Negro College Funds Special Programs (UNCFSP), grant number 003844. 


\section{References}

[1] National Council for Radiation Protection and Measurements (NCRP), Radiation protection guidance for activities in low Earth orbit. Report 132, Bethesda, Maryland (2000).

[2] National Aeronautics and Space Administration, The Vision for Space Exploration, http://www.nasa.gov/pdf/55583main_vision_space_exploration2.pdf, February (2004).

[3] J. W. Wilson, J. L. Shinn, R. K. Tripathi, R.C. Singleterry, M. S. Clowdsley, S. A. Thibeault, F. M Cheatwood, W. Schimmerling, A. K. Noor, M. Y. Kim, F. F. Badavi, J. H. Heinbockel, J. Miller, C. Zeitlin, L. Heilbronn, Issues in Deep Space Radiation Protection, Acta Astronautica, vol. 49, pp. 289 - 312 (2001).

[4] J. W. Wilson, F. F. Badavi, F. A. Cucinotta, J. L. Shinn, G. D. Badhwar, R. Silberberg, C.H. Tsao, L. W. Townsend, R. K. Tripathi, HZETRN: description of a free - space ion and nucleon transport and shielding computer program, NASA Technical Report 3495 (1995).

[5] J. W. Wilson, L. W. Townsend, W. Schimmerling, G. S. Khandelwal, F. Khan, J. E. Nealy, F. A. Cucinotta, L. C. Simonsen, J. L. Shinn, J. W. Norbury, Transport Methods and Interactions for Space Radiations. NASA Research Publication 1257 (1991).

[6] C. Amsler et al., Review of particle physics, Physics Letters B, vol. 667, pp. 1 - 1340 (2008).

[7] J. W. Wilson, R. K. Tripathi, C. J. Mertens, S. R. Blattnig, M. S. Clowdsley, F.A. Cucinotta, J. Tweed, J. H. Heinbockel, S. A. Walker, J. E. Nealy, Verification and Validation: High Charge and Energy (HZE) Transport Codes and Future Development, NASA Technical Paper 213784, (2005).

[8] K. Niita, T. Sato, H. Nakashima, H. Iwase, H. Nose, L. Sihver, PHITS: A particle and heavy ion transport code system, Radiation Measurements, vol. 41, pp. 1080 - 1090 (2006). 
[9] A. Ferrari, P.R. Sala, A. Fasso, J. Ranft, FLUKA: a multi - particle transport code (Program version 2005), CERN 2005-10, INFN/TC-05/11, SLAC-R-773 (2005).

[10] L. S. Waters, G. W. McKinney, J. W. Durkee, Michael L. Fensin, J. S. Hendricks, M. R. James, R. C. Johns, D. B. Pelowitz, The MCNPX Monte Carlo radiation transport code, AIP Conf. Proc., vol. 896, p. 81 (2007).

[11] T. A. Gabriel, B. L. Bishop, F. S. Alsmiller, R. G. Alsmiller, J. O. Johnson, CALOR95: A Monte Carlo Program Package for the Design and Analysis of Calorimeter Systems, ORNL/TM - 11185 (1995).

[12] N. V. Mokhov, S. I. Striganov, A. Van Ginneken, S. G. Mashnik, A. J. Sierk, J. Ranft, MARS Code Developments, LANL Report LA-UR-98-5716, Available at http://arxiv.org/abs/nucl-th/9812038 (1998).

[13] GEANT4, Users's Documents, Physics Reference Manual, http://wwwinfo.cern.ch/asd/ geant4/G4UsersDocuments/UsersGuides/PhysicsReferenceManual, (1998).

[14] S. K. Aghara, R. Wilkins, J. Zhou, Computational Analysis of Martian Regolith in Martian Space Environment, Space Nuclear Conference, American Nuclear Society embedded tropical meeting, San Diego (2005).

[15] S. K. Aghara, E. Wright, R. Wilkins, J. Zhou, B. Gersey, Spectral Characterization of Secondary Radiation from Regolith Materials, AIAA, Space 2007 Conference and Expo Long Beach Convention Center, CA, September (2007).

[16] T. Ersmark, P. Carlson, E. Daly, C. Fuglesang, I. Gudowska, B. Lund - Jensen, P. Nieminen, M. Pearce, G. Santin, Geant4 Monte Carlo Simulations of the Galactic Cosmic Ray Radiation Environment On - Board the International Space Station/Columbus, IEEE Transactions on Nuclear Sciences, vol. 54, no. 5, pp. 1854 - 1862 (2007). 
[17] G. W. McKinney, D. J. Lawrence, T. H. Prettyman, R. C. Elphic, W. C. Feldman, J. J. Hagerty, MCNPX benchmark for cosmic ray interactions with the Moon, Journal of Geophysical Research, vol. 111, p. E06004 (2006).

[18] L. Sihver, D. Mancusi, T. Sato, K. Niita, H. Iwase, Y. Iwamoto, N. Matsuda, H. Nakashima, Y. Sakamoto, Recent developments and benchmarking of the PHITS code, Advances in Space Research, vol. 40, pp. 1320 - 1331 (2007).

[19] J. Collot, H. G. Kirk, N. V. Mokhov, Pion production models and neutrino factories, Nuclear Instruments and Methods in Physics Research Section A, vol. 451, pp. 327 - 330 (2000).

[20] G. Battistoni, A. Ferrari, C. Forti, E. Scapparone, Simulation of muon transport at high energy: Comparison of few different codes, Nuclear Instruments and Methods in Physics A, vol. 394, pp. 136 - 145, (1997).

[21] H. Koshiishi, H. Matsumoto, A. Chishiki, T. Goka, T. Omodaka, Evaluation of the neutron radiation environment inside the International Space Station based on the Bonner Ball Neutron Detector experiment, Radiation Measurements, vol. 42, pp. 1510 - 1520 (2007).

[22] K. J. Kim, I. G. Graham, J. Masarik, R. C. Reedy, Numerical simulations with the MCNPX and LAHET Code Systems compared with direct measurement of neutron fluxes in terrestrial environments, Nuclear Instruments and Methods in Physics Research B, vol. 259, pp. 637 - 641 (2007).

[23] J. F. Briesmeister, ed., MCNP-A General Monte Carlo N-Particle Transport Code-Version 4C, Los Alamos National Laboratory report LA-13709-M (2000).

[24] J. S. Hendricks, G. W. McKinney, M. L. Fensin, M. R. James, R. C. Johns, J. W. Durkee, J. P. Finch, D. B. Pelowitz, L. S. Waters, Franz X. Gallmeier, MCNPX, VERSION 26E, LA-UR-07-6632, Nov 17, p. 70 (2007). 
[25] MCNPX 2.6.0 Manual, Los Alamos Report LA-CP-07-1473, Los Alamos, April (2008).

[26] S. G. Mashnik, A. J. Sierk, K. K. Gudima, M. I. Baznat, CEM03 and LAQGSM03-new modeling tools for nuclear applications, Journal of Physics - Conference Series, Institute of Physics, vol. 41, Issue 1, pp. 340 - 351 (2006).

[27] S. G. Mashnik, K. K. Gudima, R. E. Prael, A. J. Sierk, M. I. Baznat, N. V. Mokhov, CEM03.03 and LAQGSM03.03 Event Generators for the MCNP6, MCNPX, and MARS15 Transport Codes, Los Alamos National Laboratory Report, LA-UR-08-2931, p. 94 (2008).

[28] S. G. Mashnik et al., CEM03.01 User Manual, Los Alamos National Laboratory report, LA-UR-05-7321, (2005); RSICC Code Package PSR-532, http://wwwrsicc.ornl.gov/codes/psr/psr5/psr-532.html/ (2006).

[29] J. S. Hendricks, MCNPX Model/Table Comparison, Los Alamos National Laboratory report LA-14030 (2003).

[30] K. K. Gudima et al., Extension of the LAQGSM03.01 Code to Describe Photo-Nuclear Reactions up to Tens of GeV, Los Alamos National Laboratory report LA-UR-06-4693, p. $10(2006)$.

[31] R. E. Prael, H. Lichtenstein, User Guide to LCS: The LAHET Code System, LA-UR 893014, Radiation Transport Group, Los Alamos National Laboratory (1989).

[32] International Commission on Radiation Units and Measurements, Quantities and Units in Radiation Protection Dosimetry, International Commission on Radiation Units and Measurements, Maryland, ICRU Report, vol. 33 (1980).

[33] P. M. O'Neill, Badhwar - O'Neill galactic cosmic ray model update based on advances composition explorer (ACE) energy spectra from 1997 to present, Advances in Space Research, vol. 37, pp. 1727 - 1733 (2006). 